\title{
n-6 and n-3 Long-chain polyunsaturated fatty acids in the erythrocyte membrane of Brazilian preterm and term neonates and their mothers at delivery it $^{2}$
}

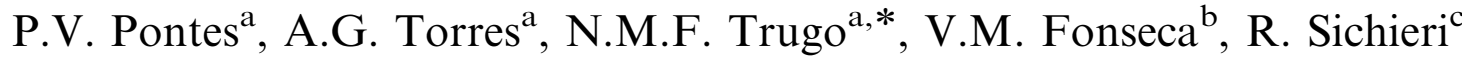 \\ ${ }^{a}$ Laboratório de Bioquímica Nutricional e de Alimentos, Instituto de Química, Universidade Federal do Rio de Janeiro, \\ Cidade Universitária, CT bl. A, 21949-900 Rio de Janeiro, RJ, Brazil \\ ${ }^{\mathrm{b}}$ Fundação Oswaldo Cruz and Universidade Federal Fluminense, RJ, Brazil \\ ${ }^{\mathrm{c}}$ Instituto de Medicina Social, Universidade do Estado do Rio de Janeiro, RJ, Brazil
}

Received 18 August 2005; received in revised form 18 October 2005; accepted 5 November 2005

\begin{abstract}
Placental transfer of the long-chain polyunsaturated fatty acids (LCPUFA) arachidonic (AA) and docosahexaenoic (DHA) acids is selectively high to maintain accretion to fetal tissues, especially the brain. The objectives of the present study were to investigate the essential fatty acid (EFA) and LCPUFA status at birth of preterm and term Brazilian infants and their mothers, from a population of characteristically low intake of n-3 LCPUFA, and to evaluate the association between fetal and maternal status, by the determination of the fatty acid composition of the erythrocyte membrane. Blood samples from umbilical cord of preterm (26-36 weeks of gestation; $n=30)$ and term (37-42 weeks of gestation; $n=30)$ infants and the corresponding maternal venous blood were collected at delivery. The LCPUFA composition of the erythrocyte membrane and DHA status were similar for mothers of preterm and term infants. Neonatal AA was higher $(P<0.01)$ whereas its precursor 18:2n-6 was lower $(P<0.01)$ than maternal levels, as expected. There was no difference in LCPUFA erythrocyte composition between preterm and term infants, except for DHA. Term infants presented a worse DHA status than preterm infants $(P<0.01)$ and than their mothers $(P<0.01)$ at delivery. There was a negative correlation of neonatal DHA with maternal AA and a positive correlation between neonatal AA and maternal AA and 18:2n-6 only at term. These results suggest that the persistent low DHA maternal status, together with the comparatively better AA and 18:2n-6 status, might have affected maternal-fetal transfer of DHA when gestation was completed up to term, and possibly contributed to the worse DHA status of term neonates compared with the preterm neonates.
\end{abstract}

(C) 2005 Elsevier Ltd. All rights reserved.

\section{Introduction}

During pregnancy, maternal, placental and fetal requirements for essential fatty acids (EFA; 18:2n-6 and 18:3n-3) and long-chain polyunsaturated fatty acids

\footnotetext{
${ }^{2}$ Sources of Support: Conselho Nacional de Desenvolvimento Científico e Tecnológico (CNPq), Coordenação de Aperfeiçoamento de Pessoal de Ensino Superior (CAPES), and Fundação Carlos Chagas Filho de Amparo à Pesquisa do Estado do Rio de Janeiro (FAPERJ), Brazil; Organización Panamericana de la Salud (OPAS).

*Corresponding author. Tel.: + 552125627352 ; fax: +552125627266

E-mail address: trugo@iq.ufrj.br (N.M.F. Trugo).
}

(LCPUFA) are high. LCPUFA, mainly arachidonic acid (AA; 20:4n-6) and docosahexaenoic acid (DHA; 22:6n-3), are indispensable functional and structural components of all cellular membranes and are especially required for fetal neural, visual and vascular development [1]. The fetus depends primarily on placental transfer of LCPUFA, which are obtained preformed from the maternal diet and/or are synthesized by the mother from EFA [1,2]. LCPUFA accretion in the fetus increases exponentially from the 30th to the 38th week of gestation [3], and therefore preterm babies may be in disadvantage compared to term babies regarding LCPUFA status $[1,4,5]$. 
The availability of LCPUFA during early life appears to affect the quality of growth and the development of visual and cognitive functions $[1,6]$. Several studies have shown that later LCPUFA status of infants is determined both by the status at birth and the post-natal diet [7-9]. Therefore, the evaluation of EFA and LCPUFA status at birth can be an important predictor of future status of fatty acids and development in infants.

EFA and LCPUFA status of newborn infants has been evaluated by the fatty acid composition of plasma phospholipids $[5,10,11]$ and of erythrocyte phospholipids $[9,12,13]$. The erythrocyte EFA and LCPUFA of infants reliably reflect maternal transfer, as has been shown by the direct correlations of these fatty acids between newborn term infants and their mothers, which are stronger than those observed in plasma phospholipids [13]. The fatty acid composition of the erythrocytes has been also used to evaluate the influence of habitual intake on maternal status of fatty acids $[7,14]$ and as a biochemical and functional index of EFA and LCPUFA maternal status [13] and infant requirements [7].

The pattern of intake of EFA and LCPUFA affects maternal [15], as well as neonatal status [16]. In Brazil, the habitual intake of lipids of the general urban population [17] and of pregnant and lactating women $[14,18,19]$ is characterized by a relatively high intake of saturated and monounsaturated fatty acids and low intake of LCPUFA, especially of the n-3 series. Therefore, it is expected that the maternal EFA and LCPUFA status resulting from this particular dietary pattern may influence fetal status. Data on maternal-fetal transfer of fatty acids in Brazilian newborn infants and the comparison between preterm and term infants regarding their LCPUFA status at birth could support future policies and guidelines, aimed at prenatal care, emphasizing the importance of LCPUFA intake during pregnancy and lactation.

The objectives of this study were to investigate the EFA and LCPUFA status of preterm and term Brazilian infants and their mothers at delivery, and to evaluate the association between fetal and maternal status, by the determination of the fatty acid composition of the erythrocyte membrane.

\section{Subjects and methods}

\subsection{Subjects}

Adult healthy pregnant women attending the Maternity Ward of the Instituto Fernandes Figueira-Fundação Oswaldo Cruz (FIOCRUZ), Rio de Janeiro, from July 2000 to May 2001 were asked to participate in the study. Exclusion criteria were maternal gestational diabetes, pregnancy-induced hypertension, metabolic or genetic disorders, infection, and complications during delivery, including a newborn with congenital malformation. A total of 140 preterm newborns and mothers were included in the period. Three days per week all mothers of term newborns were invited to participate in a total of 162. A random sub-sample of 30 pairs mother-preterm and 30 pairs mother-term newborn was chosen for the present study. The study protocol was approved by the Ethics Committee of FIOCRUZ and was conducted in accordance with the Declaration of Helsinki. All participants signed an informed consent form prior to enrolment.

Venous blood samples from 30 mothers $(27.8 \pm 7.0$ years of age, mean \pm SD) of full-term newborn infants (37-42 weeks of gestation) and from 30 mothers (26.9 \pm 8.3 years of age) of preterm infants (26-36 weeks of gestation) were collected into EDTA-containing tubes at delivery. Blood samples from the umbilical cord of the full-term and preterm newborns were also collected at delivery.

Length of gestation and infant sex, weight, length and head circumference were recorded at birth. Habitual maternal intake of food sources of fatty acids was estimated by using a food frequency questionnaire designed to collect qualitative data on sources of fat and previously validated [20]. The questionnaire was applied to the mothers within 2 days after delivery.

\subsection{Sample preparation and analysis}

Plasma was separated from erythrocytes by centrifugation, and the packed erythrocytes were washed three times with isotonic $\mathrm{NaCl}(0.9 \%, \mathrm{w} / \mathrm{v})$, after removal of the buffy coat. The washed erythrocytes were suspended in an equal volume of isotonic $\mathrm{NaCl}$ and stored at $-20{ }^{\circ} \mathrm{C}$ with sodium dithionite at a final concentration of $1.0 \%(\mathrm{w} / \mathrm{v})$ to avoid oxidation of unsaturated fatty acids [21].

Fatty acid analysis in erythrocytes was carried out by capillary gas chromatography in a Shimadzu GC-14B (Japan) gas chromatograph, using an Omegawax-320 column $(30 \mathrm{~m} \times 0.32 \mathrm{~mm}$ i.d.; Supelco, Co. $)$. Erythrocyte suspensions $(250 \mu \mathrm{L})$ were transesterified with acetyl chloride (Merck; P.A.) in methanol [22]. Methylated samples were stored in hexane $(250 \mu \mathrm{L})$ with BHT $(0.05 \%, \mathrm{w} / \mathrm{v})$ under $\mathrm{N}_{2}$ at $-20{ }^{\circ} \mathrm{C}$ for up to 1 week, until analysis. Samples $(1.5 \mu \mathrm{L})$ were injected in the gas chromatograph operated as follows: He as carrier gas $(70 \mathrm{Kpa})$; detector temperature $=280^{\circ} \mathrm{C}$; injector temperature $=240^{\circ} \mathrm{C}$; split ratio $=1: 20$; column temperature gradient $=170{ }^{\circ} \mathrm{C}(5 \mathrm{~min})+2{ }^{\circ} \mathrm{C} / \mathrm{min}$ up to $210^{\circ} \mathrm{C}(20 \mathrm{~min})$. The fatty acid methyl esters were identified by comparison of relative retention times with authentic standards ("Menhaden oil"; 37 FAME mix; Supelco, Co). Results are expressed as \% (by weight) of total fatty acids. 
To characterize the EFA and DHA status of the mothers and of their newborn infants, the following indices were calculated: EFA status index (EFASTI) was obtained by dividing the sum of the essential $n-3$ and $n-6$ fatty acids by the total amount of the nonessential n-7 and n-6 fatty acids; DHA sufficiency index (DHASI) was calculated as the ratio $22: 6 n-3 / 22: 5 n-6$; and the DHA deficiency index (DHADI) was calculated as the ratio $22: 5 n-6 / 22: 4 n-6$ [13].

\subsection{Statistical analyses}

Data were analyzed with Statgraphics v.4.0 (Statistical Graphics Corporation/Plus Ware, USA). Variables with non-normal distribution were normalized with log transformation before analyses. For statistical comparison between groups the Student's $t$ tests (paired $t$ test for comparison between mothers and neonates; unpaired $t$ test for comparison between term and preterm neonates and between their mothers) were used. Pearson correlation analysis was used to investigate associations between maternal and neonatal fatty acids and between characteristics of the newborn infants (gestational age, weight, length and head circumference at birth) and PUFA. Two-tailed $P$ values $<0.05$ were considered statistically significant.

\section{Results}

Table 1 shows the general characteristics of the newborn infants. The expected differences between preterm and term neonates were observed.

Fig. 1 shows the frequency of intake of fat sources by the mothers participating in the study. The intake of fat sources was similar in mothers of preterm and term neonates, and therefore the frequency of intake is shown together for all mothers. Vegetable oils, mainly soyabean oil $(>90 \%)$, were consumed daily by $100 \%$ of these mothers, and were the main source of EFAs, especially $18: 2 \mathrm{n}-6$. Hydrogenated vegetable fat, which is the main ingredient of margarine and a rich source of saturated fatty acids and trans-monounsaturated fatty

Table 1

Characteristics of the newborn infants

\begin{tabular}{lcc}
\hline & Preterm $(n=30)$ & Term $(n=30)$ \\
\hline Gestational age (weeks) & $33 \pm 2.4^{\mathrm{a}}$ & $39 \pm 1.3$ \\
Birth weight (g) & $2142 \pm 660^{\mathrm{a}}$ & $3149 \pm 322$ \\
Birth length (cm) & $44.8 \pm 4.0^{\mathrm{a}}$ & $49.9 \pm 2.5$ \\
Head circumference $(\mathrm{cm})$ & $31.5 \pm 3.1^{\mathrm{a}}$ & $34.6 \pm 1.3$ \\
\hline
\end{tabular}

Results are presented as mean $\pm \mathrm{SD}$.

${ }^{\mathrm{a}}$ Significantly different from term neonates (Student's $t$ test): $P<0.001$.

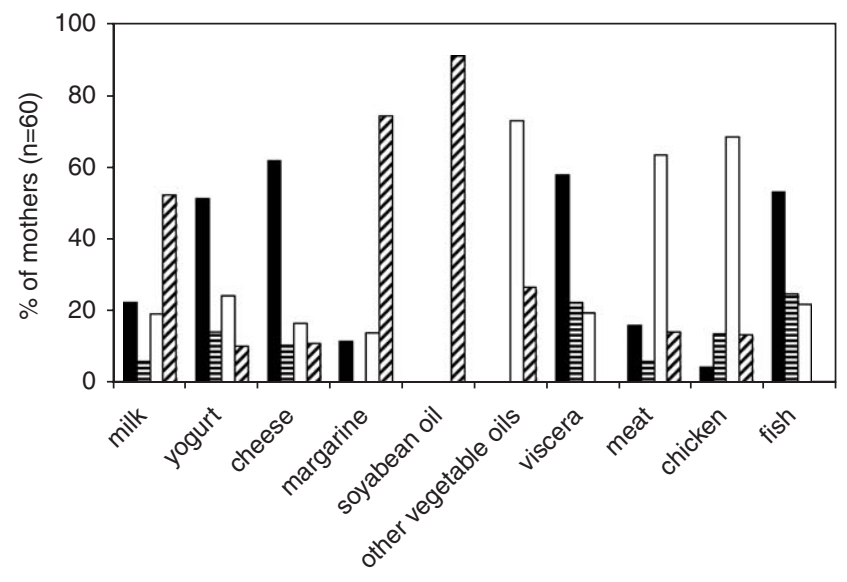

Fig. 1. Frequency of intake of fat sources in the maternal diet: never; 目 monthly; $\square$ weekly; 贯 daily.

acids was consumed daily by $75 \%$ of the mothers. Sixtyfour and $69 \%$ of the mothers consumed weekly meat and chicken, respectively. These foods are the main sources of saturated fatty acids and AA in the western diet. Approximately $53 \%$ of the mothers consumed milk daily, which was the main dairy food consumed. Approximately $50 \%$ of the mothers reported that fish was never consumed, $20 \%$ consumed once a month and $20 \%$ once a week, showing a low intake of a major source of n-3 LCPUFA.

The fatty acid composition of the erythrocyte membrane of preterm and term neonates and their mothers is reported in Table 2. Saturated fatty acids comprised the majority of the fatty acids in the erythrocyte membrane, varying from $50 \%$ to $58 \%$. PUFA concentrations ranged from $30 \%$ to $34 \%$, and those of the n-6 series, mainly AA and 18:2n-6, were the most abundant PUFA. Total n-3 PUFA was 5-7\%, with DHA being the most abundant (approximately $4.0 \%$ ). There were no significant differences in PUFA composition between mothers of preterm and term neonates, except for 18:2n-6 and 18:4n-3, which were, respectively, lower and higher $(P<0.01)$ in mothers of preterm neonates. As for the newborn infants, significant differences $(P<0.01)$ were found for DHA and the total n-3 fatty acids, which were lower in term neonates than in preterm neonates. EFASTI, DHADI and DHASI values did not differ significantly between mothers of preterm and term infants, but term neonates presented a lower $(P<0.01)$ DHASI value than preterm neonates. When we compared the fatty acid profile of preterm neonates of 26-32 weeks of gestation $(n=7)$ with that of the preterm neonates of 33-36 weeks ( $n=23 ; t$-test), we found no significant difference between them. Correlation analysis of fatty acids and weeks of gestation also did not show any significant association. Therefore, we reported the results for all preterm neonates (26-36 weeks) in Table 2. 
Table 2

Fatty acid composition ( $\%$ of total fatty acids) of erythrocyte membrane of preterm and term newborn infants and their mothers at delivery

\begin{tabular}{|c|c|c|c|c|}
\hline \multirow[t]{2}{*}{ Fatty acids } & \multicolumn{2}{|l|}{ Term } & \multicolumn{2}{|l|}{ Preterm } \\
\hline & $\begin{array}{l}\text { Mothers } \\
(n=30)\end{array}$ & $\begin{array}{l}\text { Infants } \\
(n=30)\end{array}$ & $\begin{array}{l}\text { Mothers } \\
(n=30)\end{array}$ & $\begin{array}{l}\text { Infants } \\
(n=30)\end{array}$ \\
\hline \multicolumn{5}{|l|}{ Saturated } \\
\hline $16: 0$ & $25.1 \pm 2.3$ & $27.6 \pm 2.1^{\mathrm{a}}$ & $25.3 \pm 4.0$ & $28.7 \pm 5.1^{\mathrm{a}}$ \\
\hline 18:0 & $16.0 \pm 1.4$ & $19.2 \pm 1.4^{\mathrm{a}}$ & $16.5 \pm 2.7$ & $19.4 \pm 3.4^{\mathrm{a}}$ \\
\hline $20: 0$ & $0.4 \pm 0.1$ & $0.8 \pm 0.1^{\mathrm{a}}$ & $0.5 \pm 0.1$ & $0.7 \pm 0.2^{\mathrm{a}}$ \\
\hline $22: 0$ & $2.2 \pm 0.4$ & $1.9 \pm 0.5^{\mathrm{a}}$ & $2.3 \pm 0.5$ & $1.8 \pm 0.4^{\mathrm{a}}$ \\
\hline $24: 0$ & $6.1 \pm 1.2$ & $6.7 \pm 1.6$ & $6.6 \pm 1.0$ & $7.1 \pm 1.4$ \\
\hline \multicolumn{5}{|c|}{ Monounsaturated } \\
\hline $16: \ln -7$ & $0.5 \pm 0.2$ & $0.6 \pm 0.2$ & $0.6 \pm 0.3$ & $0.7 \pm 0.4$ \\
\hline $18: 1 n-7$ & $2.2 \pm 0.5$ & $1.9 \pm 0.3^{\mathrm{a}}$ & $2.5 \pm 0.7$ & $2.1 \pm 0.4^{\mathrm{a}, \mathrm{b}}$ \\
\hline $18: 1 n-9$ & $10.8 \pm 0.8$ & $8.5 \pm 1.2^{\mathrm{a}}$ & $10.9 \pm 0.9$ & $9.0 \pm 1.5^{\mathrm{a}}$ \\
\hline $24: 1 \mathrm{n}-9$ & $3.6 \pm 0.9$ & $4.1 \pm 1.3$ & $3.7 \pm 0.9$ & $3.4 \pm 1.7$ \\
\hline \multicolumn{5}{|c|}{ Polyunsaturated } \\
\hline $18: 2 n-6$ & $9.8 \pm 1.6$ & $3.5 \pm 0.9^{\mathrm{a}}$ & $8.4 \pm 2.2^{\mathrm{c}}$ & $3.3 \pm 1.0^{\mathrm{a}}$ \\
\hline $18: 4 n-3$ & $0.8 \pm 0.4$ & $1.0 \pm 0.5$ & $1.2 \pm 0.5^{\mathrm{c}}$ & $1.0 \pm 0.4$ \\
\hline $20: 3 n-6$ & $1.6 \pm 0.3$ & $2.3 \pm 0.8^{\mathrm{a}}$ & $1.6 \pm 0.4$ & $2.0 \pm 0.8^{\mathrm{a}}$ \\
\hline $20: 4 n-6$ & $11.4 \pm 2.2$ & $13.7 \pm 3.7^{\mathrm{a}}$ & $11 \pm 3.6$ & $12.7 \pm 5.9^{\mathrm{a}}$ \\
\hline $22: 4 n-6$ & $3.0 \pm 0.5$ & $3.1 \pm 0.9$ & $3.2 \pm 0.9$ & $2.7 \pm 1.1$ \\
\hline $22: 5 n-6$ & $0.9 \pm 0.3$ & $1.4 \pm 0.4^{\mathrm{a}}$ & $1.0 \pm 0.3$ & $1.1 \pm 0.3$ \\
\hline $22: 5 n-3$ & $1.4 \pm 0.2$ & $0.5 \pm 0.4^{\mathrm{a}}$ & $1.4 \pm 0.4$ & $0.6 \pm 0.7^{\mathrm{a}}$ \\
\hline $22: 6 n-3$ & $4.6 \pm 0.5$ & $4.0 \pm 0.7^{\mathrm{a}}$ & $4.6 \pm 0.4$ & $4.5 \pm 0.6^{\mathrm{b}}$ \\
\hline Total n-6 & $27.2 \pm 0.2$ & $25.4 \pm 0.3^{\mathrm{a}}$ & $27.2 \pm 0.3$ & $26.2 \pm 0.4$ \\
\hline Total n-3 & $6.8 \pm 0.1$ & $5.2 \pm 0.2^{\mathrm{a}}$ & $7.0 \pm 0$ & $6.0 \pm 0.1^{\mathrm{a}, \mathrm{b}}$ \\
\hline $\mathrm{n} 3 / \mathrm{n} 6$ ratio & $0.25 \pm 0.02$ & $0.20 \pm 0.03^{\mathrm{a}}$ & $0.26 \pm 0.02$ & $0.22 \pm 0.04^{\mathrm{a}}$ \\
\hline EFASTI & $2.0 \pm 0.2$ & $2.1 \pm 0.2$ & $2.0 \pm 0.2$ & $2.1 \pm 0.2$ \\
\hline DHADI & $0.3 \pm 0.1$ & $0.5 \pm 0.2^{\mathrm{a}}$ & $0.3 \pm 0.1$ & $0.4 \pm 0.1^{\mathrm{a}}$ \\
\hline DHASI & $5.8 \pm 1.8$ & $2.8 \pm 0.8^{\mathrm{a}}$ & $5.1 \pm 1.6$ & $4.0 \pm 1.0^{\mathrm{a}, \mathrm{b}}$ \\
\hline
\end{tabular}

Results are presented as mean \pm SD. $n 3 / n 6$ : ratio between the sum of all n-3 and n-6 fatty acids; DHADI: DHA deficiency index (22:5n-6 22:4n-6); DHASI: DHA sufficiency index (22:6n-3/22:5n-6); EFASTI: EFA status index $(\Sigma \mathrm{n}-6+\Sigma \mathrm{n}-3 / \Sigma \mathrm{n}-7+\Sigma \mathrm{n}-9)$. The 20:1n-9 and 20:5n-3 fatty acids are present in trace amounts. Variables with nonsymmetrical distributions $(16: 1 n-7 ; 18: 2 n-6 ; 20: 4 n-6 ; 22: 4 n-6 ; 22: 5 n-6$; $22: 5 n-3 ; 24: 1 n-9)$ were log transformed before analysis.

${ }^{a}$ Significantly different from mothers $(P<0.05)$.

${ }^{\mathrm{b}}$ Significantly different from term neonates $(P<0.01)$

${ }^{\mathrm{c}}$ Significantly different from term mothers $(P<0.01)$.

Comparison of the erythrocyte fatty acid composition between the newborn infants and their mothers (Table 2) showed that there were significant differences for saturated, monounsaturated and PUFA. Among the latter, $18: 2 n-6,22: 5 n-3$, total $n-3, n 3 / n 6$ ratio and DHASI in both preterm and term neonates, and 22:6n-3 only in term neonates, were lower $(P<0.05)$ than maternal levels. On the other hand, 20:3n-6, 20:4n-6 and DHADI in both preterm and term neonates, and $22: 5 \mathrm{n}-6$ and total $\mathrm{n}-6$ only in term neonates, were higher $(P<0.05)$ than maternal levels. There was no difference in EFASTI between mothers and newborn infants.
Significant correlations between several PUFA in maternal and in neonatal erythrocytes are shown in Table 3. In both preterm and term neonates and in their mothers there were positive correlations between PUFA precursor-product of n-6 fatty acids. Negative correlations were found in neonates in both groups between 18:0 and n-6 PUFA. These PUFA also presented negative correlations with 18:1n-9 in neonates in both groups and in mothers of preterm infants only. For all groups there were negative correlations between LCPUFA of $n-6$ and $n-3$ series.

Table 4 shows the significant correlations of PUFA between the newborn infants and their mothers. A significant positive correlation was found between AA in term infants and maternal AA and its precursor 18:2n-6, whereas DHA contents in term infants presented a negative correlation with maternal AA and its elongation product $22: 4 n-6$. In preterm infants the only direct significant correlations were with maternal $22: 5 n-6$ and DHASI, but there was also a negative correlation between neonatal DHASI and maternal DHADI. In addition, maternal $20: 3 \mathrm{n}-6$ tended $(P=0.06)$ to correlate with preterm 20:3n-6 $(r=0.36)$ and $22: 5 n-3$ $(r=-0.47)$.

Pearson's correlation analysis between erythrocyte fatty acids and length of gestation, weight at birth and length at birth of preterm and term infants showed associations between weight at birth and $18: 1 \mathrm{n}-9$ $(r=0.42 ; P=0.02)$ and $18: 1 \mathrm{n}-7(r=0.36 ; P=0.05)$ only for the term neonates, whom also presented a positive correlation between length at birth and 18:2n-6 $(r=0.40 ; P=0.03)$.

\section{Discussion}

The pattern of intake of food fat sources in the present study was similar to that found for Brazilian women in other studies [14,18,19] and showed a high maternal intake of vegetable oils, mainly soya-bean oil, and a low intake of fish and fish oils. This dietary pattern results, respectively, in high intake of $18: 2 n-6$ and low intake of preformed n-3 LCPUFA, which were reflected in the maternal LCPUFA status in our study. In comparison with mothers from the Netherlands [13], where the intake of n-3 fatty acids is higher [23], Brazilian mothers presented a worse DHA status at delivery, as shown by their lower DHASI and higher DHADI values in the erythrocyte membrane, whereas AA levels were similar. EFASTI values were apparently higher in the Brazilian mothers, due mainly to the contribution of 18:2n-6 and AA. Comparison with German mothers [24] also showed that Brazilian mothers had higher levels of erythrocyte 18:2n-6 plus AA. 
Table 3

Significant Pearson correlations between PUFA in neonatal erythrocytes and between PUFA in maternal erythrocytes

\begin{tabular}{|c|c|c|c|c|c|}
\hline Neonatal erythrocytes & $r$ & $P$ & Maternal erythrocytes & $r$ & $P$ \\
\hline Term $(n=30)$ & & & Term $(n=30)$ & & \\
\hline $18: 0$ vs. $20: 4 n-6$ & -0.68 & 0.035 & $18: 0$ vs. $18: 2 n-6$ & -0.70 & 0.001 \\
\hline $18: 1 n-9$ vs. $20: 4 n-6$ & -0.63 & 0.032 & $18: 0$ vs. $20: 4 n-6$ & -0.68 & 0.001 \\
\hline $20: 3 n-6$ vs. $20: 4 n-6$ & 0.80 & 0.001 & $18: 2 n-6$ vs. $20: 4 n-6$ & 0.85 & 0.014 \\
\hline $20: 4 n-6$ vs. $22: 6 n-3$ & -0.52 & 0.005 & $18: 2 n-6$ vs. $22: 6 n-3$ & -0.37 & 0.049 \\
\hline $22: 4 n-6$ vs. $22: 6 n-3$ & -0.37 & 0.043 & $20: 4 n-6$ vs. $22: 6 n-3$ & -0.35 & 0.048 \\
\hline Preterm $(n=30)$ & & & Preterm $(n=30)$ & & \\
\hline $18: 0$ vs. $18: 2 n-6$ & -0.80 & 0.001 & $18: 0$ vs. $18: 2 n-6$ & -0.86 & 0.001 \\
\hline $18: 0$ vs. $20: 4 n-6$ & -0.94 & 0.001 & $18: 1 n-9$ vs. $18: 2 n-6$ & -0.45 & 0.037 \\
\hline $18: 1 n-9$ vs. $20: 4 n-6$ & -0.44 & 0.015 & $18: 1 n-9$ vs. $20: 4 n-6$ & -0.54 & 0.004 \\
\hline $18: 2 n-6$ vs. $20: 4 n-6$ & 0.67 & 0.005 & $18: 2 n-6$ vs. $20: 4 n-6$ & 0.84 & 0.001 \\
\hline $18: 2 n-6$ vs. $22: 6 n-3$ & -0.82 & 0.001 & $20: 3 n-6$ vs. $20: 4 n-6$ & 0.66 & 0.005 \\
\hline $20: 3 n-6$ vs. $20: 4 n-6$ & 0.90 & 0.001 & & & \\
\hline $20: 4 n-6$ vs. $22: 6 n-3$ & -0.59 & 0.004 & & & \\
\hline
\end{tabular}

Table 4

Significant Pearson correlations between neonatal and maternal erythrocyte PUFA

\begin{tabular}{|c|c|c|c|c|c|c|}
\hline \multirow[t]{2}{*}{ Mothers PUFA } & \multicolumn{3}{|c|}{ Term neonates $(n=30)$} & \multicolumn{3}{|c|}{ Preterm neonates $(n=30)$} \\
\hline & PUFA & $R$ & $P$ & PUFA & $r$ & $P$ \\
\hline $18: 2 n-6$ & $20: 4 n-6$ & 0.50 & 0.0047 & & - & - \\
\hline $20: 4 n-6$ & $20: 4 n-6$ & 0.77 & 0.0001 & - & - & - \\
\hline $20: 4 n-6$ & $22: 4 n-6$ & 0.42 & 0.0478 & - & - & - \\
\hline $22: 4 n-6$ & $22: 5 n-6$ & 0.60 & 0.0076 & - & - & - \\
\hline $20: 4 n-6$ & $22: 6 n-3$ & -0.44 & 0.0157 & - & - & - \\
\hline $22: 4 n-6$ & $22: 6 n-3$ & -0.51 & 0.0029 & - & - & - \\
\hline $22: 5 n-6$ & - & - & - & $22: 5 n-6$ & 0.57 & 0.0177 \\
\hline DHADI & - & - & - & DHASI & -0.58 & 0.0141 \\
\hline DHASI & - & - & - & DHASI & 0.67 & 0.0031 \\
\hline
\end{tabular}

DHADI: DHA deficiency index (22:5n-6/22:4n-6); DHASI: DHA sufficiency index (22:6n-3/22:5n-6).

The fatty acid composition of the maternal blood largely determines the fatty acid mix delivered to the fetus, but placental transfer is selectively higher for AA and DHA than for other PUFA. This transfer involves a multistep process of selective uptake by the placental microvillous membrane, intracellular channeling of individual fatty acids and selective export to the fetal circulation [2]. Fatty acids cross the placental microvillous membrane by simple diffusion and via the action of a membrane bound fatty-acid binding protein, which presents higher affinities and binding capacities for AA and DHA than for other fatty acids [25-27]. Accordingly, studies using erythrocyte membrane $[12,13,28]$ and plasma phospholipids $[5,28,29]$ in the cord blood have shown that AA and DHA relative contents ( $\mathrm{g} \%)$ are higher, whereas 18:2n-6 is lower in newborn infants than in their mothers, and that full-term infant levels of EFA and LCPUFA are related to maternal levels [13]. In the present study, we found similar results for the differences between maternal and fetal relative levels of
EFA and LCPUFA, except for DHA. In contrast with the studies mentioned [12,13,28], our study showed similar erythrocyte DHA contents in preterm infants and their mothers and lower erythrocyte DHA in term infants compared to their mothers.

Comparison between term and preterm Brazilian infants showed that preterm infants were not in disadvantage in regard to EFA and LCPUFA status. On the contrary, term infants presented lower DHA and DHASI levels in the erythrocyte membrane than preterm infants, despite the similarity in maternal DHA status in both groups. Furthermore, term Brazilian infants had lower DHASI and higher DHADI than term infants from the Netherlands [13].

Studies in pregnant women $[3,13,30]$ have shown that there is a progressive decline in EFA, AA and DHA maternal status during the last 12 weeks of pregnancy, and that at delivery DHASI is lower and DHADI is higher in full-term infants than in their mothers [13]. Although AA, DHA, DHASI, DHADI and EFASTI 
were not different between mothers of term and preterm infants in the present study, the imbalance between the low DHA maternal status and the relatively adequate 18:2n-6 and AA status possibly contributed to a low fetal DHA status, which was more perceptible at the end of term gestation. This hypothesis is consistent with the negative association between DHA in term newborn infants and maternal AA, the positive association between $\mathrm{AA}$ in these infants and maternal AA and $18: 2 n-6$, and the lack of these correlations in the preterm infants in the present study. It is also worth noting that in neonatal erythrocytes there was a negative correlation between AA and DHA, and in mothers of term infants there were negative correlations between $18: 2 n-6$ and DHA and between AA and DHA (Table 3), in contrast to the highly significant positive correlations between erythrocyte AA and DHA observed in pregnant British and Korean mothers and in their term babies at birth [31].

Taken together these results suggest that the current levels of intake of n-3 LCPUFA by Brazilian pregnant women, characteristic of the Brazilian urban population, may not be sufficient to sustain an increased maternal-fetal transfer of DHA until term. EFA metabolism and conversion to LCPUFA is extremely competitive and 18:3n-3 conversion to DHA is dependent on the relative abundance of 18:2n-6 [32], therefore the evaluation of n-3 and n-6 fatty acid maternal status should be considered simultaneously. The persistent low DHA maternal status, together with the comparatively better AA and 18:2n-6 status, might have affected maternal-fetal transfer of DHA when gestation was completed up to term, and possibly contributed to the worse DHA status of term neonates compared with the preterm neonates.

\section{Acknowledgements}

The authors thank the women who volunteered to participate in this study. PVP is a recipient of a Ph.D. fellowship (CNPq), and NMFT and RS are Research Fellows of CNPq.

\section{References}

[1] M. Crawford, Placental delivery of arachidonic and docosahexaenoic acids: implications for the lipid nutrition of preterm infants, Am. J. Clin. Nutr. 71 (Suppl. 1) (2000) 275S-284S.

[2] P. Haggarty, Placental regulation of fatty acid delivery and its effect on fetal growth - a review, Placenta 23 (Suppl. A) (2002) S28-S38.

[3] A.C. van Houwelingen, G. Hornstra, MDM Al, Long-chain polyunsaturated fatty acids, pregnancy, and pregnancy outcome, Am. J. Clin. Nutr. 71 (Suppl.) (2000) 285S-291S.

[4] M.M. Foreman-van Drongelen, A.C. van Houwelingen, A.D. Kester, T.H. Hasaart, C.E. Blanco, G. Hornstra, Long-chain polyunsaturated fatty acids in preterm infants: Status at birth and its influence on postnatal levels, J. Pediatr. 126 (1995) 611-618.

[5] S.L. Elias, S.M. Innis, Infant plasma trans, n-6, and n-3 fatty acids and conjugated linoleic acids are related to maternal plasma fatty acids, length of gestation, and birth weight and length, Am. J. Clin. Nutr. 73 (2001) 807-814.

[6] R. Uauy, P. Mena, C. Rojas, Essential fatty acids in early life: structural and functional role, Proc. Nutr. Soc. 59 (2000) 3-15.

[7] R.A. Henderson, R.G. Jensen, C.J. Lammi-Keefe, A.M. Ferris, K.R. Dardick, Effect of fish oil on the fatty acid composition of human milk and maternal and infant erythrocytes, Lipids 27 (1992) 863-869.

[8] M. Makrides, M.A. Neumann, Effect of maternal docosahexaenoic acid (DHA) supplementation on breast milk composition, Eur. J. Clin. Nutr. 50 (1996) 352-357.

[9] P. Guesnet, P. Pugo-Gunsam, C. Maurage, M. Pinault, B. Giraudeau, J.M. Alessandri, et al., Blood lipid concentrations of docosahexaenoic and arachidonic acids at birth determine their relative postnatal changes in term infants fed breast milk or formula, Am. J. Clin. Nutr. 70 (1999) 292-298.

[10] T.M. Berghaus, H. Demmelmair, B. Koletzko, Fatty acid composition of lipid classes in maternal and cord plasma at birth, Eur. J. Pediatr. 157 (1998) 763-768.

[11] P. Rump, R.P. Mensink, A.D.M. Kester, G. Hornstra, Essential fatty acid composition of plasma phospholipids and birth weight: a study in term neonates, Am. J. Clin. Nutr. 73 (2001) 797-806.

[12] M. Ruyle, W.E. Connor, G.J. Anderson, R.I. Lowensohn, Placental transfer of essential fatty acids in humans: venousarterial difference for docosahexaenoic acid in fetal umbilical erythrocytes, Proc. Natl. Acad. Sci. USA 87 (1990) 7902-7906.

[13] H. Vlaardingerbroek, G. Hornstra, Essential fatty acids in erythrocyte phospholipids during pregnancy and at delivery in mothers and their neonates: comparison with plasma phospholipids, Prostaglandins Leukot. Essent. Fatty Acids 71 (2004) 363-374.

[14] A.G. Torres, J.G. Ney, F. Meneses, N.M.F. Trugo, Polyunsaturated fatty acids and conjugated linoleic acid isomers in breast milk are associated with plasma nonesterified and erythrocyte membrane fatty acid composition in lactating women, Br. J. Nutr. (2006) in press.

[15] S.M. Innis, S.L. Elias, Intakes of essential n-6 and n-3 polyunsaturated fatty acids among pregnant Canadian women, Am. J. Clin. Nutr. 77 (2003) 473-478.

[16] A.C. van Houwelingen, J.D. Sorensen, G. Hornstra, M.M Simonis, J. Boris, S.F. Olsen, N.J. Secher, Essential fatty acid status in neonates alter fish-oil supplementation during late pregnancy, Br. J. Nutr. 74 (1995) 723-731.

[17] C.A. Monteiro, L. Mondini, R.B.L. Costa, Mudanças na composição e adequação da dieta familiar nas áreas metropolitanas do Brasil (1988-1996), Rev. Saúde Públ. 34 (2000) 251-258.

[18] J. da Cunha, T.H.M. da Costa, M.K. Ito, Influences of maternal dietary intake and suckling on breast milk lipid and fatty acid composition in low-income women from Brasilia, Brazil, Early Hum. Dev. 81 (2005) 303-311.

[19] M.H.L. Silva, M.T.C. Silva, S.C.C. Brandão, J.C. Gomes, L.A. Peternelli, S.C.C. Franceschini, Fatty acid composition of mature breast milk in Brazilian women, Food Chem. 93 (2005) 297-303.

[20] R. Sichieri, J.E. Everhart, Validity of a Brazilian food frequency questionnaire against dietary recalls and estimated energy intake, Nutr. Res. 18 (1998) 1649-1659.

[21] R.M. Broekhuyse, Long-term storage of erythrocytes for quantitative analyses of lipids, Clin. Chim. Acta 52 (1974) 53-58.

[22] G. Lepage, C.C. Roy, Direct transesterification of all classes of lipids in a one-step reaction, J. Lipid Res. 27 (1986) 114-120.

[23] Zo eet Nederland, Resultaten van de Voedselconsumptiepeiling 1997-1998, Voedingscentrum, Den Haag, 1998. 
[24] H. Böhles, S. Arndt, U. Ohlenschläger, T. Beeg, B. Gebhardt, A.C. Sewell, Maternal plasma homocysteine, placenta status and docosahexaenoic acid concentration in erythrocytes phospholipids of the newborn, Eur. J. Pediatr. 158 (1999) 243-246.

[25] P. Haggarty, K. Page, D.R. Abramovich, J. Ashton, D. Brown, Long-chain polyunsaturated fatty acid transport across the perfused human placenta, Placenta 18 (1997) 635-642.

[26] F.M. Campbell, P.G. Bush, J.H. Veerkamp, A.K. Dutta-Roy, Detection and cellular localization of plasma membrane-associated and cytoplasmic fatty acid-binding proteins in human placenta, Placenta 19 (1998) 409-415.

[27] A.K. Dutta-Roy, Transport mechanisms for long-chain polyunsaturated fatty acids in the human placenta, Am. J. Clin. Nutr. 71 (2000) 315S-322S.

[28] Y. Min, K. Ghebremeskel, M.A. Crawford, J.H. Nam, A. Kim, I.S. Lee, H. Suzuki, Maternal-fetal n-6 and n-3 polyunsaturated fatty acids gradient in plasma and red cell phospholipids, Int. J. Vitamin Nutr. Res. 71 (2001) 286-292.
[29] M.A. Crawford, K. Costeloe, K. Ghebremeskel, A. Phylactos, L. Skirvin, F. Stacey, Are deficits of arachidonic and docosahexaenoic acids responsible for the neural and vascular complications of preterm babies?, Am. J. Clin. Nutr. 66 (Suppl. 4) (1997) 1032S-1041S.

[30] G. Hornstra, M.D. Al, A.C. van Houwelingen, M.M. Foremanvan Drongelen, Essential fatty acids in pregnancy and early human development, Eur. J. Obstet. Gynecol. Reprod. Biol. 61 (1995) 57-62.

[31] K. Ghebremeskel, Y. Min, M.A. Crawford, J.H. Nam, A. Kim, J.N. Koo, H. Suzuki, Blood fatty acid composition of pregnant and nonpregnant Korean women: red cells may act as a reservoir of arachidonic acid and docosahexaenoic acid for utilization by the developing fetus, Lipids 35 (2000) 567-574.

[32] S.M. Innis, Plasma and red blood cell fatty acid values as indexes of essential fatty acids in the developing organs of infants fed with milk or formulas, J. Pediatr. 120 (1992) S78-S86. 